\title{
Hepatic artery aneurysms: Endovascular therapeutic techniques
}

\author{
Umberto Geremia Rossi ${ }^{1}$ and Francesco Petrocelli ${ }^{2}$ \\ ${ }^{1}$ Department of Radiological Area, Interventional Radiology Unit, E.O. Galliera Hospital, \\ ${ }^{2}$ Department of Radiology and Interventional Radiology, IRCCS San Martino Policlinic University Hospital, \\ Genova, Italy
}

\begin{abstract}
Hepatic Artery Aneurysm (HAA) is a rare disease, but it can be a life-threatening pathology if it is ruptured. MultiDetector Computed Tomography has to be considered the "gold standard" diagnostic imaging in detecting HAA and it is essential for treatment planning. Treatment for HAA can be surgical or endovascular. Endovascular approaches in HAA, compare to conventional abdominal surgery, benefit in less invasive treatments. The aim of our paper is to emphasize the three possible endovascular therapeutic techniques in HAA: packing embolization, isolation embolization and stenting deployment. (Ann Hepatobiliary Pancreat Surg 2021;25:167-170)
\end{abstract}

Key Words: Hepatic Artery; Stent; Aneurysm; Embolization; Endovascular; Imagin

\section{Dear Sir,}

we appreciated the paper of Rosenberg et al. ${ }^{1}$ entitled: "Management of hepatic artery aneurysm: a case series".

The authors have described a series of four Hepatic Artery Aneurysm (HAA) cases that were all diagnosed by Multi-Detector Computed Tomography (MD-CT). One patient was treated by endovascular stenting, the other was candidate to follow-up and the last two treated by surgery techniques.

HAA is a rare disease, but can result fatal if it is ruptured. ${ }^{1-3}$ We agree with the use of MD-CT as "gold standard" diagnostic imaging in detecting HAA and for capable of treatment planning. ${ }^{1,4}$ Treatment of HAA is indicate in case of: clinical symptoms (pain, distal embolization, rupture), twice the diameter of the hepatic artery or more than $2 \mathrm{~cm}$ increased in diameter, and in pregnant women. $^{1-3}$ Invasive treatment for HAA can be surgical or endovascular. Endovascular approaches in HAA, compare to conventional abdominal surgery, benefit in less invasive treatments. ${ }^{2}$ Aim of our editorial is to emphasize the three possible endovascular therapeutic techniques in HAA (common and proper hepatic artery segments - ruptured and unruptured): i) packing embolization, ii) iso- lation embolization and iii) stenting deployment.

i) Packing embolization technique (or direct embolization technique) is characterized by coil or liquid agent device filling of the aneurysm sac. ${ }^{4}$ To guarantee patency of the hepatic artery segment and to prevent possible coil migration out of the aneurysm's sac it is possible to place a bare stent into the artery segment (stent assisted technique). ${ }^{5}$ However, this technique provides a limit in subsequent imaging follow-up, mainly with MD-CT. On MD-CT especially coils or high-density liquid embolic devices create artefacts, which may not highlight a possible endoleak at the level of the treated HAA (Fig. 1). In these cases colour-Doppler ultrasound, contrast-enhanced ultrasound, or angiography are used to follow-up treated aneurysm over time.

ii) Isolation embolization technique (or sandwich technique) is characterized by the complete embolization of all outflow (distal) and inflow (proximal) arteries of the aneurysm sac by coil and/or plug devices. ${ }^{1,4}$ This technique is not always feasible on anatomical variants of collateral vessels. However, when performed, in the subsequent MD-CT follow-up there are present only few artefacts are present that allow evaluating aneurysm thrombo-

Received: September 24, 2020; Revised: November 27, 2020; Accepted: November 27, 2020

Corresponding author: Umberto Geremia Rossi

Department of Radiological Area, Interventional Radiology Unit, E.O. Galliera Hospital, Mura delle Cappuccine, 14, Genova 16128, Italy Tel: +39-010-563-4152, Fax: +39-010-563-4152, E-mail: urossi76@hotmail.com

Copyright (C) 2021 by The Korean Association of Hepato-Biliary-Pancreatic Surgery

This is an Open Access article distributed under the terms of the Creative Commons Attribution Non-Commercial License (http://creativecommons.org/ licenses/by-nc/4.0) which permits unrestricted non-commercial use, distribution, and reproduction in any medium, provided the original work is properly cited. Annals of Hepato-Biliary-Pancreatic Surgery • pISSN: 2508-5778 - elSSN: 2508-5859 

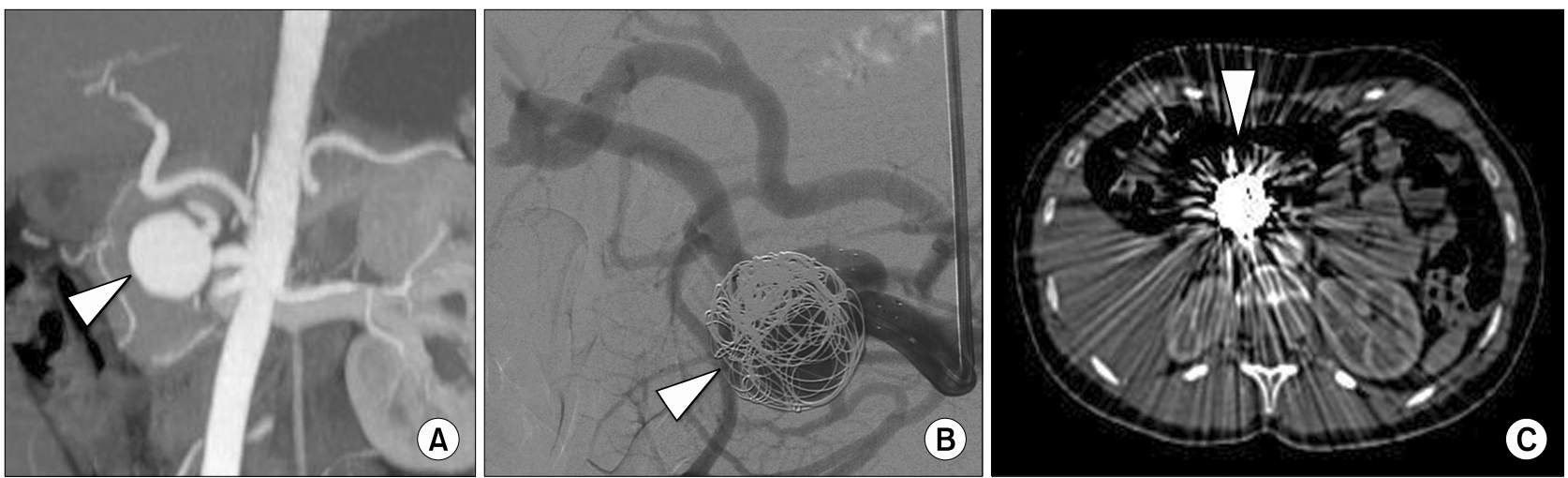

Fig. 1. (A) Contrast enhanced Multi-Detector Computed Tomography coronal MIP reconstruction image of the upper abdomen that shows a saccular right hepatic artery aneurysm of $3 \mathrm{~cm}$ in diameter (arrowhead). (B) Selective right hepatic artery Digital Subtraction Angiography during coil devices deployment into the aneurysm sac (arrowhead). (C) Contrast enhanced MultiDetector Computed Tomography axial image at 6-months follow-up that confirms the presence of coils into the right hepatic artery aneurism (arrowhead) with multiple metallic artefact.

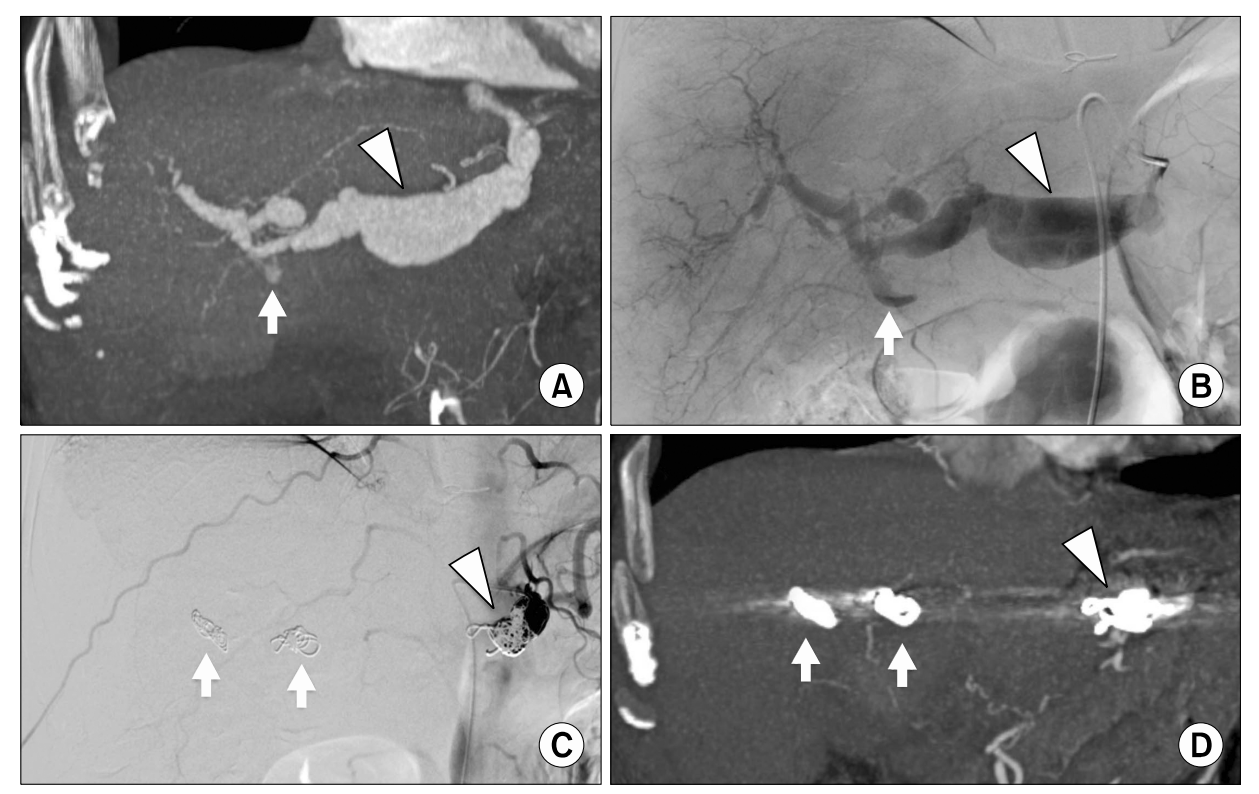

Fig. 2. (A) Contrast enhanced Multi-Detector Computed Tomography coronal MIP reconstruction image of the upper abdomen that shows a hepatic artery fusiform aneurysm of $3.7 \mathrm{~cm}$ in diameter (arrowhead), a focal wall tear with contrast media extravasation as sign of rupture (arrow) and dilatation of the right and left hepatic arteries. (B) Selective hepatic artery Digital Subtraction Angiography that confirms the hepatic artery fusiform aneurysm (arrowhead) and the focal wall tear with contrast media extravasation as sign of rupture (arrow). (C) Final selective hepatic artery Digital Subtraction Angiography that confirms the exclusion of hepatic artery fusiform aneurysm with isolation embolization technique by coils deployment into right and left hepatic arteries (arrows) and into post-origin of common hepatica artery (arrowhead). (D) Contrast enhanced Multi-Detector Computed Tomography coronal MPR reconstruction image of the upper abdomen at 12-months follow-up that confirms the exclusion of hepatic artery fusiform aneurysm with coils artefacts at the level of right and left hepatic arteries (arrows) and post-origin of common hepatica artery (arrowhead).

sis or endoleak (Fig. 2). Isolation embolization technique is equivalent to surgical exclusion ligation. The main disadvantage of this technique is that it does not preserve the patency of the treated artery segment. There is a very low risk of possible ischemic lesion of the liver due to its dual vascularization (portal vein approximately $70 \%$ and hepatic artery $30 \%$ of liver blood flow) and an artery vascular compensation over time by collateral vessels. ${ }^{2-4}$

iii) Stenting deployment technique, using covered or flow-diverter devices, has the aim of guarantee aneurysm 


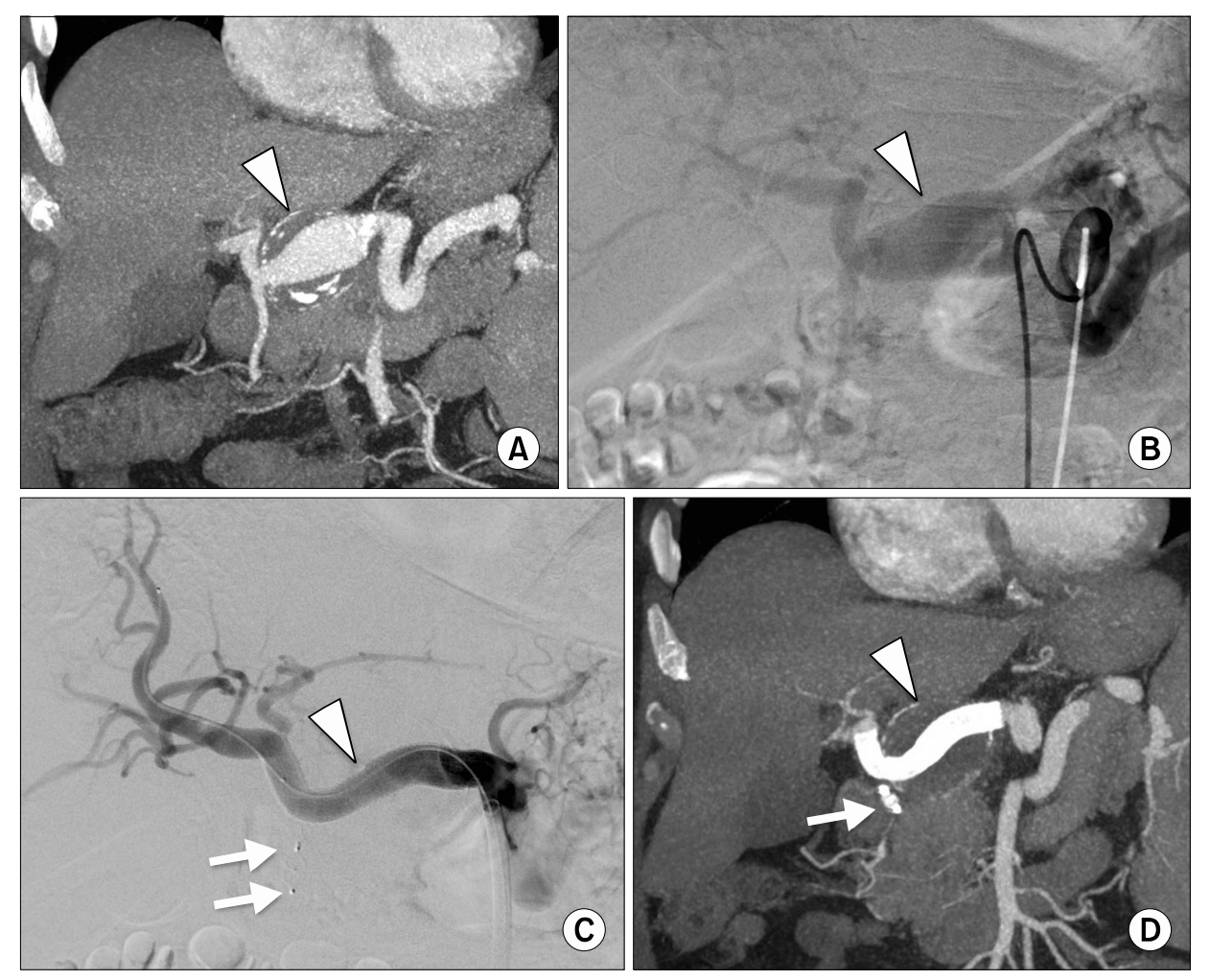

Fig. 3. (A) Contrast enhanced Multi-Detector Computed Tomography coronal MIP reconstruction image of the upper abdomen that shows a common hepatic artery aneurysm of $3.8 \mathrm{~cm}$ in diameter with partial thrombosis and wall calcifications (arrowhead), (B) Selective hepatic artery Digital Subtraction Angiography that confirms common hepatic artery aneurysm (arrowhead). (C) Final selective hepatic artery Digital Subtraction Angiography that confirms the exclusion of common hepatic artery aneurysm by covered stent deployment (arrowhead) with distal artery patency; note the two metal artefact of plug device deployed at the origin of gastroduodenal artery (arrows). (D) Contrast enhanced Multi-Detector Computed Tomography coronal MPR reconstruction image of the upper abdomen at 24-months follow-up that confirms the exclusion common hepatic artery aneurysm by covered stent deployment (arrowhead) and metal artefact at gastroduodenal artery origin due to plug device (arrow).

exclusion and to ensure blood perfusion to distal hepatic branch vessels. This type of endovascular approach is often limited by aneurysm location (need for distal and proximal neck) and arterial anatomy (tortuosity that can limit the navigability of rigid and large-calibre stents) (Fig. 3). Flow-diverter stents may be possible to maintain patency of collateral vessel of the aneurysm by modulating intra-sac blood flow; ${ }^{2,3}$ but they are not indicated in pseudo or ruptured HAAs. In conclusion, in patients affected by HAA an appropriate multidisciplinary discussion focusing attention on aneurysm anatomical location, characteristics, extension, hemodynamic status, patient age and comorbidities are necessary to determine the specific treatment for each individual case.

\section{CONFLICT OF INTEREST}

Neither author has any conflicts of interest, financial or otherwise, relating to the content here.

\section{ORCID}

\author{
Umberto Geremia Rossi: \\ https://orcid.org/0000-0001-9785-4237 \\ Francesco Petrocelli: \\ https://orcid.org/0000-0001-9841-0355
}

\section{AUTHOR CONTRIBUTIONS}

Conceptualization: UGR. Formal analysis: UGR, FP. Methodology: UGR, FP. Project administration: UGR. Visualization: UGR, FP. Writings: UGR. Writing - review \& editing: FP. 


\section{REFERENCES}

1. Rosenberg A, Trebska-McGowan K, Reichman T, Sharma A, Cotterell A, Strife B, et al. Management of hepatic artery aneurysm: a case series. Ann Hepatobiliary Pancreat Surg 2020;24:333338.

2. Obara H, Kentaro M, Inoue M, Kitagawa Y. Current management strategies for visceral artery aneurysms: an overview. Surg Today 2020;50:38-49.
3. Chiaradia M, Novelli L, Deux JF, Tacher V, Mayer J, You K, et al. Ruptured visceral artery aneurysms. Diagn Interv Imaging 2015;96:797-806.

4. Ferro C, Rossi UG, Bovio G, Petrocelli F, Seitun S. The Amplatzer vascular plug 4: preliminary experience. Cardiovasc Intervent Radiol 2010;33:844-848.

5. Ferro C, Rossi UG, Seitun S, Bovio G, Castellan L, De Paolis $\mathrm{M}$, et al. Hepatic arterial loop with accessory right hepatic artery aneurysm with celiac atresia: endovascular therapy with a stent and detachable coils. J Vasc Interv Radiol 2008;19:1236-1240. 